\title{
Utility as a User Selection Criterion for Coordinated Multi-Point Systems
}

\author{
Annika Klockar*, Carmen Botella ${ }^{\dagger}$, Mikael Sternad ${ }^{\ddagger}$, Anna Brunstrom*, Tommy Svensson ${ }^{\S}$ \\ *Computer Science, Karlstad University, Karlstad, Sweden, \{annika.klockar, anna.brunstrom\}@kau.se \\ $\dagger$ IRTIC, Universitat de València, València, Spain, carmen.botella@uv.es \\ ${ }^{\ddagger}$ Signals and Systems, Uppsala University, Uppsala, Sweden, mikael.sternad@ signal.uu.se \\ $\S$ Signals and Systems, Chalmers University of Technology, Gothenburg, Sweden, tommy.svensson@chalmers.se
}

\begin{abstract}
Coordinated multipoint (CoMP) transmission and reception techniques have been proposed to combat inter-cell interference in cellular systems and, hence, to increase the data rates. Due to the overhead introduced, not all users may be served with CoMP. In this paper, we focus on how to select users for joint processing CoMP under limited backhaul capacity. The evaluated user selection schemes take user experience into account, quantified by the utility of Internet application types. We propose a heuristic algorithm utility based user selection that has low computational complexity. Utility based user selection and maximize utility, which maximizes the sum utilities, are compared to maximize rate, which maximizes the sum data rates. A range of traffic mixes and user locations are evaluated. The simulation results indicate that resources required for CoMP are more efficiently used if the user selection is based on utility rather than on maximizing the total data rate. If utility is taken into account a higher total utility can in many cases be achieved for the same limited backhaul capacity.
\end{abstract}

\section{INTRODUCTION}

The number of users in cellular networks and the data traffic volume have increased significantly over the last years. The introduction of smartphones and tablets have lead to an increasing part of data traffic that requires high data rates, such as online video. The capacity in cellular networks is more limited than in wired networks, in theory by the Shannon limit, and, in practice, even more by the current spectrum regulations and the deployed technologies. Network resources could be more efficiently used if user experience was taken into account when resources are allocated. To quantify user experience, the utility of Internet application types can be used. Utility is often used to quantify different levels of user satisfaction and to address decision problems in networking, such as resource allocation [1] and network selection for vertical handover [2]. The utility of an application depends on the application performance, which in turn depends on the Quality of Service (QoS) offered by the system [3].

In cellular systems, inter-cell interference is a limiting factor for providing the high data rates required for satisfactory QoS, in particular at the cell-edges. Coordinated multipoint transmission/reception (CoMP) techniques are intended to reduce inter-cell interference with joint processing/transmission or coordinated scheduling/beamforming [4]. Under ideal conditions, joint processing can remove inter-cell interference completely [5]. However, overhead is introduced in terms of required channel knowledge at the transmitter side, inter base station (BS) information exchange, and feedback from the users. System wide joint processing is not feasible in practice, since the overhead introduced would be too large. The amount of user data and control information that is possible to exchange between BSs depends on the capacity of the backhaul links. From the results of field trials and simulations presented in [6], the authors conclude that the backhaul technologies currently used in LTE are, in principle, capable of supporting CoMP, but that the amount of data exchanged between the BSs needs to be restricted.

In this paper, we reduce the load on backhaul links by selecting a subset of the users for joint processing CoMP. The radio resource management (RRM) decisions on user selection are assumed to be taken on relatively long time scales, in the order of 0.5s [7]. Admission control is not in the scope of this paper, but is assumed as a prior step performed before the user selection. The main focus of this paper is user selection, and, therefore, scheduling on a resource block level is not in the scope either. We evaluate user selection schemes that take user experience into account. To our knowledge, application utility has not previously been evaluated for user selection for CoMP. Previous work on CoMP under limited backhaul capacity typically focus on maximizing sum rate per cell, see [5] and references therein.

Our previous results obtained under unlimited backhaul capacity, presented in [8], indicate that the impact of joint processing CoMP on user experience is highly dependent on the application type. In this paper, we investigate the gain of using application utility when users are selected for joint processing CoMP. Two schemes based on utility are evaluated, maximize utility and utility based user selection. Maximize utility has the objective to maximize the total utility of the users' application utilities. As the computational complexity of utility maximization is high, we propose utility based user selection, which is a heuristic algorithm of low computational complexity. The two schemes are compared to maximize rate, a scheme that has the objective to maximize the sum of the users' rates. The simulation results indicate that the total utility is higher for maximize utility and utility based user selection than for maximize rate, since the resources required for CoMP are more efficiently used. On average the total utility is $15.9-17.6 \%$ higher, but for individual traffic mixes and backhaul capacities the increase is over $40 \%$. If also individual 


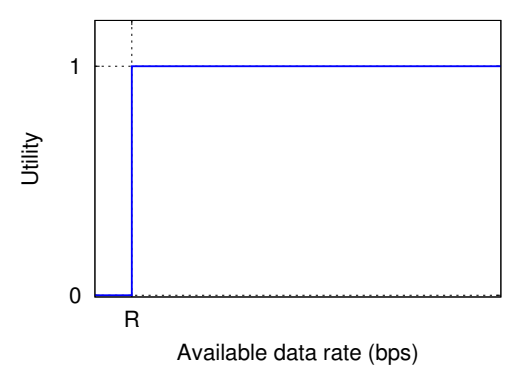

(a)

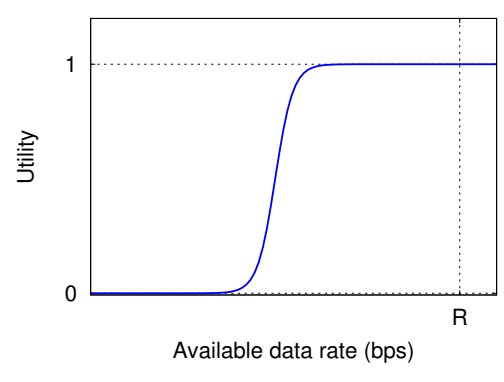

(b)

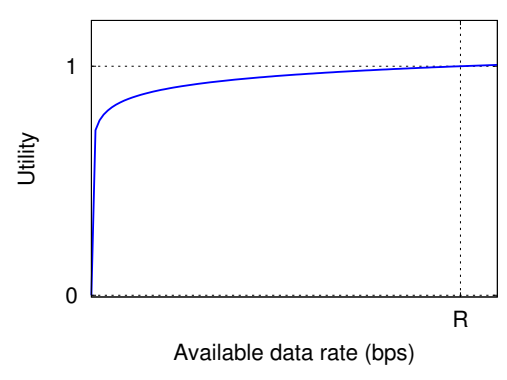

(c)

Fig. 1. Utility of application types: (a) hard real-time, (b) adaptive and (c) elastic

shadow fading values are considered, then the total utility may be twice as high.

The rest of the paper is structured as follows. In Section II, application types and their utility functions are described. An overview of the system model is given in Section III. User selection is discussed in Section IV. In Section V, the simulation setup and parameter values are presented. The simulation results are summarized and discussed in Section VI. The paper is concluded in Section VII.

\section{APPLICATION TYPES AND THEIR UTILITY FUNCTIONS}

We consider three utility functions for application types that are frequently used in the Internet: hard real-time, adaptive, and elastic applications [3]. The specific utility functions used in this paper are equivalent to three of the utility functions in [1]. We selected the utility functions and the parameter settings in order to represent application types with very different characteristics. The utility functions represent the users' satisfaction level of the data rate at which an application is allowed to transmit. Other metrics, such as delay, delay jitter and loss rate, are also used, but less often than data rate. In practice, the utility functions should, of course, be further tuned to specific application types and to the economic over-all goals.

Hard real-time applications, such as speech, require a constant data rate at a certain level, which is illustrated by the utility function in Fig. 1a. For hard real-time applications, the following utility function is used

$$
u(r)=\frac{\operatorname{sgn}(r-R)+1}{2},
$$

where $r$ is the available data rate and $R$ is the data rate required for the maximum utility $u_{R}=1$.

The utility function for adaptive applications, such as streaming media and on-line gaming, shown in Fig. 1b, is similar to the one for hard real-time applications, but smoother. The utility function used for adaptive applications is

$$
u(r)=\frac{1}{1+(1 / \epsilon-1)^{(1-2 r / R)}},
$$

where $R$ is the data rate required for the maximum utility $u_{R}=1-\epsilon$. In this paper, we evaluate an adaptive utility function with $\epsilon=10^{-9}$, which gives a function that clearly

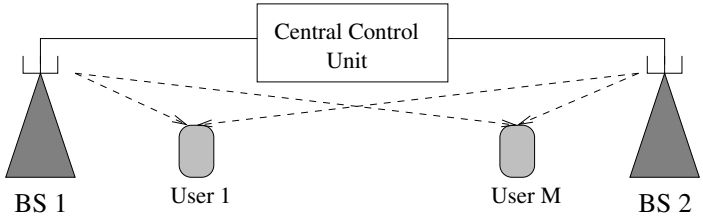

Fig. 2. Linear cellular system

differs from the ones used for hard real-time and elastic applications.

For elastic applications, such as file transfer and e-mail, the higher the data rate the better, but, as shown in Fig. 1c, the utility gain is higher at low data rates. Logarithm functions are commonly used for the utility function of elastic applications [1], [3]. The following utility function is used for elastic applications

$$
u(r)=\frac{\ln (r+1)}{\ln (R+1)},
$$

where $R$ is the data rate required for $u_{R}=1$. Elastic applications can, in contrast to hard real-time and adaptive applications, use an even higher data rate, but with only marginally increased utility. The value of $R$ could, for example, illustrate the maximum data rate that a user pays for.

\section{SYSTEM MODEL}

We consider the downlink of a linear cellular system of $K=2$ BSs, as illustrated in Fig. 2. Each BS is equipped with $N_{t}$ transmit antennas. There are $M$ single antenna users in the system. All users are served at the same time with single BS transmission by multi-user zero-forcing MIMO transmission. Selected users are also served with joint processing CoMP, over an additional bandwidth, in addition to single BS transmission. Users are selected for CoMP, as described below in Section IV. The linear precoding scheme using zero-forcing proposed in [9] is applied on the transmitter side. Strong synchronization mechanisms are required to ensure coherent reception at the user. A central control unit, located in an additional network node or in one of the BSs, is assumed to perform joint processing based on global channel state information (CSI). User data is exchanged between the BSs and the central control unit over backhaul links which have 
a certain capacity, $C$, which, for simplicity, is represented by the maximum number of users that can be served with joint processing.

Let $\mathcal{M}$ denote the set of all $M$ users, $\mathcal{M}_{\mathrm{s}}$ the set of users served with single BS transmission and $\mathcal{M}_{\mathrm{j}}$ the set of users served with CoMP. As all users are served with single BS transmission $\mathcal{M}_{\mathrm{s}}=\mathcal{M}$. Selected users are also served with CoMP which implies that $\emptyset \subseteq\left(\mathcal{M}_{\mathrm{s}} \cap \mathcal{M}_{\mathrm{j}}\right) \subseteq \mathcal{M}$. Two orthogonal frequency blocks are assumed. One of the frequency blocks is used for single BS transmission and the other for CoMP. Let $B_{\text {tot }}$ denote the total bandwidth in the system. Then, the bandwidth allowed for CoMP is $B_{\mathrm{j}}=B_{\mathrm{tot}}(C / M)$, and the bandwidth used for single BS transmission is $B_{\mathrm{s}}=B_{\mathrm{tot}}-B_{\mathrm{j}}$. A user that is served in a frequency block is allocated the full bandwidth in that frequency block. For single BS transmission, the maximum number of users that can be served in each cell is $N_{t}$, and in total $\left|\mathcal{M}_{\mathrm{s}}\right| \leq K N_{t}$ users can be served in $B_{\mathrm{s}}$ by both BSs. In $B_{\mathrm{j}}$, the number of users served with CoMP by both BSs is determined by $\left|\mathcal{M}_{\mathrm{j}}\right| \leq C \leq K N_{t}$.

The data rate of a user served with single BS transmission is

$$
r_{\mathrm{s}, m}=B_{\mathrm{s}} \log _{2}\left(1+\mathrm{SINR}_{\mathrm{s}, m}\right),
$$

and the user data rate obtained with CoMP is

$$
r_{\mathrm{j}, m}= \begin{cases}B_{\mathrm{j}} \log _{2}\left(1+\mathrm{SINR}_{\mathrm{j}, m}\right) & \text { for } m \in \mathcal{M}_{\mathrm{j}} \\ 0 & \text { for } m \notin \mathcal{M}_{\mathrm{j}} .\end{cases}
$$

The resulting data rate for a user is

$$
r_{m}=r_{\mathrm{s}, m}+r_{\mathrm{j}, m} .
$$

Flat fading is here assumed over the total bandwidth $B_{\text {tot }}$. The SINR of user $m \in \mathcal{M}_{\mathrm{x}}$, where $x=\{\mathrm{s}, \mathrm{j}\}$ is

$$
\operatorname{SINR}_{\mathbf{x}, m}=\frac{\left|\boldsymbol{h}_{m}^{T} \boldsymbol{w}_{m}\right|^{2} p_{m}}{\sum_{n \in \mathcal{M}_{x}, n \neq m}\left|\boldsymbol{h}_{m}^{T} \boldsymbol{w}_{n}\right|^{2} p_{n}+\sigma^{2}},
$$

where, in the single BS transmission case, i.e., for $m \in \mathcal{M}_{\mathrm{s}}$, $\boldsymbol{h}_{m}^{T} \in \mathbb{C}^{1 \times N_{t}}$ represents the channels between the $m$ th user and its serving BSs and $\boldsymbol{w}_{m} \in \mathbb{C}^{N_{t} \times 1}$ are the beamforming weights [9] related to the serving BS. In the CoMP case, i.e., for $m \in \mathcal{M}_{\mathrm{j}}, \boldsymbol{h}_{m}^{T} \in \mathbb{C}^{1 \times K N_{t}}$ represents the channels between the $m$ th user and the $K$ BSs and $\boldsymbol{w}_{m} \in \mathbb{C}^{K N_{t} \times 1}$ are the beamforming weights [9] related to both BSs. The power spectral density allocated to the user across the $K$ BSs is $p_{m}$ and $\sigma^{2}$ is the noise spectral density. The transmission power spectral density at each BS is assumed to be limited by a maximum value, $P_{\max }$, and equal user power allocation as in [9], is applied within each frequency block. This implies that $p_{m}=p_{n}$, for all $m, n$. To fulfill this constraint, the power spectral density allocation matrix $\mathbf{P}=\operatorname{diag}\left\{p_{j}\right\}$ is scaled as

$$
\sqrt{\mathbf{P}}=\left\{\min _{k=1, \cdots, K} \sqrt{\frac{P_{\max }}{\left\|\mathbf{W}^{(k)}\right\|_{F}^{2}}}\right\} \cdot \mathbf{I}_{[M \times M]},
$$

where $\mathbf{W}^{(k)}$ is the beamforming weights of the $k$ th $\mathrm{BS},(\cdot)_{F}$ the Frobenius norm, and $\mathbf{I}_{[M \times M]}$ an identity matrix of size

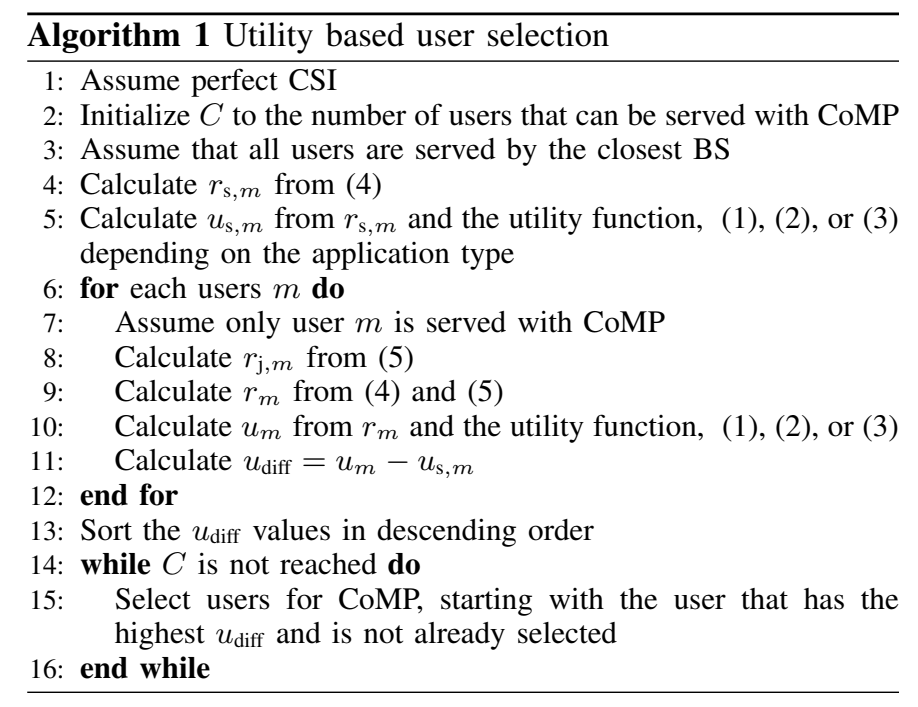

$[M \times M]$. More advanced power allocation is not examined, since it is not in the scope of this paper. The evaluation metrics used in this paper are total utility $\sum_{m=1}^{M} u_{m}\left(r_{m}\right)$ and for comparison also total rate $\sum_{m=1}^{M} r_{m}$.

\section{USER SELECTION}

User selection is applied for CoMP in order to reduce the information exchange over the backhaul links. The main focus of this paper is to evaluate what can be gained by taking application utility into account when users are selected.

We evaluate a scheme that takes application utility into account, maximize utility,

$$
\begin{array}{ll}
\operatorname{maximize} & \sum_{m=1}^{M} u_{m}\left(r_{m}\right) \\
\text { subject to } & \left|\mathcal{M}_{j}\right| \leq C
\end{array}
$$

and, compare it to a rate based scheme, maximize rate,

$$
\begin{array}{ll}
\text { maximize } & \sum_{m=1}^{M} r_{m} \\
\text { subject to } & \left|\mathcal{M}_{j}\right| \leq C .
\end{array}
$$

For mixed traffic, it is hard to find optimal algorithms that are efficient for maximizing utility, since the utility functions of adaptive applications are nonconcave and hard real-time applications have discontinuous utility functions [1], [10]. The problem is further complicated due to CoMP, since for precoding schemes that cancel interference, such as zeroforcing, which is used in this paper, the achievable data rate available to a user is highly dependent on the set of selected users [7].

Maximize utility and maximize rate perform an exhaustive search over all combinations of user selections, which gives a computational complexity of $O\left(2^{M}\right)$. As the computational complexity of maximize utility is high, we also evaluate a heuristic algorithm, utility based user selection, described in 
Algorithm 1. The utility based user selection algorithm selects the user that would gain the most from CoMP until $C$ is reached or all users are selected. The algorithm produces suboptimal results, but the complexity is much lower than for maximize utility, since only the case when one user is served with CoMP is used in the calculations. As the gains of CoMP, the $u_{\text {diff }}$ values, are sorted the computational complexity becomes $O(M \log M)$. Although the utility based user selection algorithm only considers the gain of individual users and not the gain user sets, the results in Section VI suggest an acceptable trade-off between results and complexity.

\section{Simulation SETUP}

The simulation setup consists of $K=2 \mathrm{BSs}$, each one equipped with an array of $N_{t}=3$ antennas. The cell radius is 500 meters. There are $M=6$ single antenna users. The users are served in two orthogonal frequency blocks, as described in Section III. The total bandwidth that is divided between the frequency blocks is $B_{\mathrm{tot}}=1 \mathrm{MHz}$. The channel coefficients between the $m$ th user and the $k$ th BS are modeled as $\boldsymbol{h}_{m k}=$ $\boldsymbol{h}^{\prime}{ }_{m k} \sqrt{\gamma_{s} \gamma_{p}}$, where the shadow fading is a random variable described by a log-normal distribution, $\gamma_{s} \sim \mathcal{N}(0,8 d B)$, the pathloss follows the 3GPP Long Term Evolution (LTE) model, $\gamma_{p}[d B]=148.1+37.6 \log _{10}\left(d_{m k}\right)$, where $d_{m k}$ is the distance between the $k$ th BS and the $m$ th user, and $\boldsymbol{h}_{m k}^{\prime}$ includes the small scale fading coefficients, which are i.i.d. complex Gaussian values according to $\mathcal{C N}(0,1)$. Flat fading channels are assumed. The system SNR, the average SNR received at the cell edge, has been set to $15 \mathrm{~dB}$, which implies an interference limited system.

In a CoMP enabled system, user selection for joint processing is assumed to be performed less often than scheduling decisions, say every $0.5 \mathrm{~s}$ [7]. To capture this assumption to some extent, we evaluate maximize utility and maximize rate for 12 sets of shadow fading values. For each shadow fading set, the user data rates are evaluated and averaged over 30 channel realizations with different small scale fading values, and the application utilities are calculated from the data rates.

Two scenarios are simulated, one with the users spread out between the BSs and another with all users at the cell edge. In the first scenario, illustrated in Fig. 3, the users are spread out with 3 users in each cell along the line between the BSs, with $125 \mathrm{~m}$ between the users, and between the first user and the BS. The second scenario is shown in Fig. 4. Here, the users are placed 3 in each cell within $30 \mathrm{~m}$ from the cell edge along the line between the BSs. The simulated traffic mixes are shown in Table I. Each user is assumed to run one hard real-time, adaptive, or elastic application. The traffic mixes represent some variation in application types and the locations of the users, and are sample subsets of the possible traffic mixes. The users are labeled according to their relative distances to BS 1 along the line between BSs, as shown in Fig. 3 and Fig. 4. In the case of single BS transmission, BS 1 serves users 1 to 3 and BS 2 users 4 to 6 with zero-forcing beamforming within cells. The $R$ values were chosen to represent a system
TABLE I

TRAFFIC MIXES: LABELS OF USERS

\begin{tabular}{|l|l|l|l|}
\cline { 2 - 4 } \multicolumn{1}{c|}{} & $\begin{array}{l}\text { Hard real-time } \\
\mathrm{R}=1 \mathrm{Mbps}\end{array}$ & $\begin{array}{l}\text { Adaptive } \\
\mathrm{R}=10 \mathrm{Mbps}\end{array}$ & $\begin{array}{l}\text { Elastic } \\
\mathrm{R}=10 \mathrm{Mbps}\end{array}$ \\
\hline Traffic mix 1 & 3,6 & 2,5 & 1,4 \\
\hline Traffic mix 2 & 3,5 & 1 & $2,4,6$ \\
\hline Traffic mix 3 & 3,5 & $2,4,6$ & 1 \\
\hline Traffic mix 4 & \multicolumn{2}{|c}{} \\
\hline
\end{tabular}

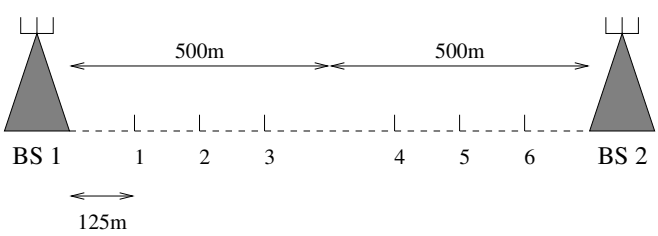

Fig. 3. User locations for users spread out between the BSs

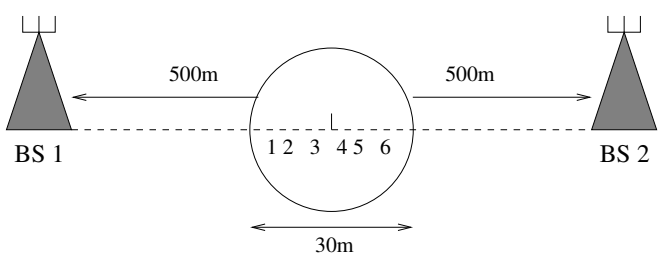

Fig. 4. User locations for all users at the cell edge

in which not all users can be satisfied with only single BS transmission.

\section{Simulation RESUlTS}

In this section, the simulation results of maximize utility, utility based user selection, and maximize rate are described and compared. The schemes are evaluated with respect to total utility and total data rate. For comparison purpose, we have also included the case when all users are served with single BS transmission, $\left|\mathcal{M}_{\mathrm{s}}\right|=M$ and $\mathcal{M}_{\mathrm{j}}=\emptyset$. We also define single $B S$ extra resources as a scheme in which the same users as the ones selected by the maximize utility scheme use the additional bandwidth $B_{\mathrm{j}}$, but use single BS transmission (which creates interference) also in this band. The purpose of generating these results is to isolate the utility gains obtained by allocating extra bandwidth to some users from the additional gains obtained by also using CoMP in that band. The backhaul capacity, $C$, is varied from no capacity at all, which corresponds to all users being served by the serving BS only, and to a capacity high enough to allow all users to be served with CoMP by both BSs.

\section{A. Users spread out between the BSs}

In the first set of simulations, the users are spread out as shown in Fig. 3. This implies that when all users are served only by their serving BS, the average data rate differs significantly between the users located close to one of the BSs and the users located further away. In Fig. 5, the total utility of all users, averaged over the 12 simulated shadow fading 


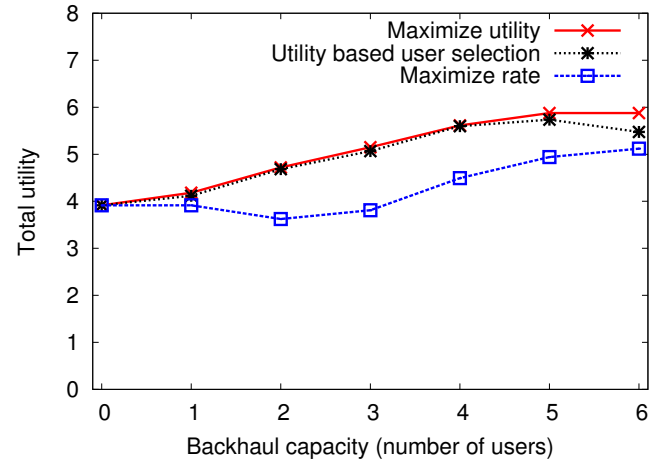

Fig. 5. Total utility for traffic mix 1 vs. backhaul capacity, users spread out between the BSs

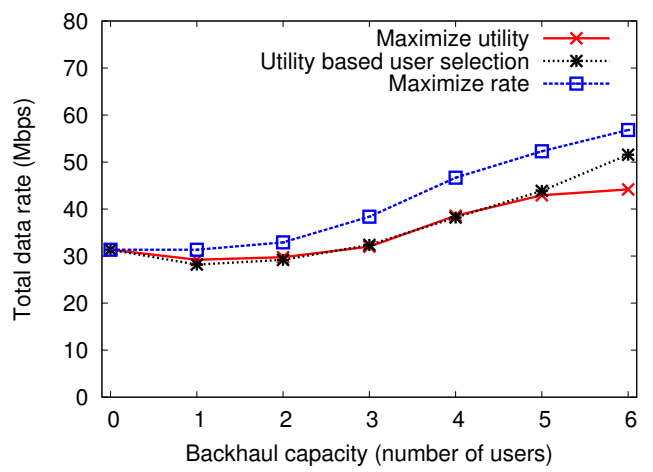

Fig. 6. Total data rate for traffic mix 1 vs. backhaul capacity, users spread out between the BSs

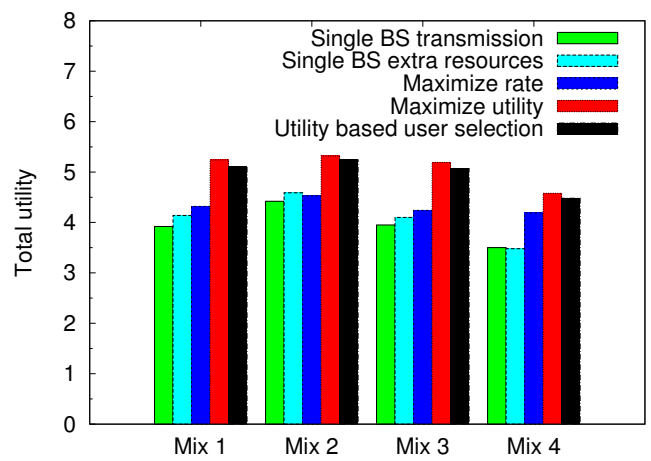

Fig. 7. Avg. over backhaul capacities of the total utility, users spread out between the BSs

sets, is shown for traffic mix 1 . The $x$-axis indicates backhaul capacity. The total utility is higher for maximize utility and utility based user selection than for maximize rate. However, the total data rate, illustrated in Fig. 6, is higher for maximize rate.

In Fig. 7, the average results over the shadow fading sets and the backhaul capacities are presented for the traffic mixes from Table I. Single BS transmission represents the case when all users are served only by the serving BS, which is the result for backhaul capacity $C=0$ in the figures. The results for

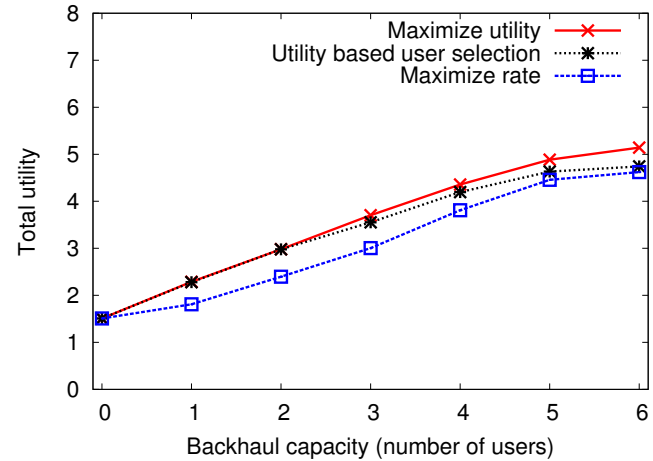

Fig. 8. Total utility for traffic mix 1 vs. backhaul capacity, all users at the cell edge

maximize utility, utility based user selection, and maximize rate are averaged over $1 \leq C \leq 6$, since these are the cases when users can be selected for CoMP.

For the evaluated traffic mixes, a higher average total utility is reached for maximize utility and utility based user selection than for maximize rate. In average over all the traffic mixes the increase of maximize utility over maximize rate is $17.6 \%$. For most user locations and shadow fading values, the utility of hard real-time applications reaches the maximum utility already with single BS transmission and also for elastic applications high utilities are often reached. Maximize utility, therefore, selects users of adaptive applications more often than applications of other traffic types. The increase is highest for traffic mix $3,22.2 \%$, since this is the traffic mix with the highest amount of adaptive and hard real-time applications. The amount of hard real-time applications has the largest impact, which can be concluded from the fact that the increase is lowest for traffic mix 4, 9.4\%, which lacks hard real-time applications. The total utility may increase even more, with $43.6 \%$, for individual traffic mixes and backhaul capacities. If also individual shadow fading sets are considered, then gains of $82.2 \%$ are observed. The increase in total utility does, however, not come from a higher data rate, but from sharing the available resources more efficiently from a utility perspective. The results for single BS extra resources are slightly better than for single BS transmission, but not in the range of the results for CoMP with the utility based schemes.

\section{B. All users at the cell edge}

In the second set of simulations, all users are located close to the cell edge, as shown in Fig. 4. When all users are served only by their serving BS, the average data rate is approximately the same for all users. The total utility and the total data rate for traffic mix 1 , averaged over the 12 shadow fading sets, are shown in Fig. 8, and Fig. 9, respectively. The total utility is higher for maximize utility and utility based user selection and the total data rate is lower than for maximize rate. As all the users are located at the cell edge, the achievable data rates are lower, than in the first set of simulations with the users spread out between the BSs. 


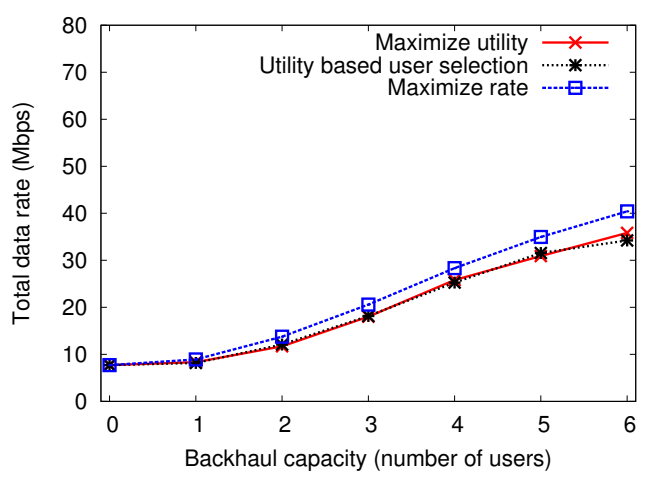

Fig. 9. Total data rate for traffic mix 1 vs. backhaul capacity, all users at the cell edge

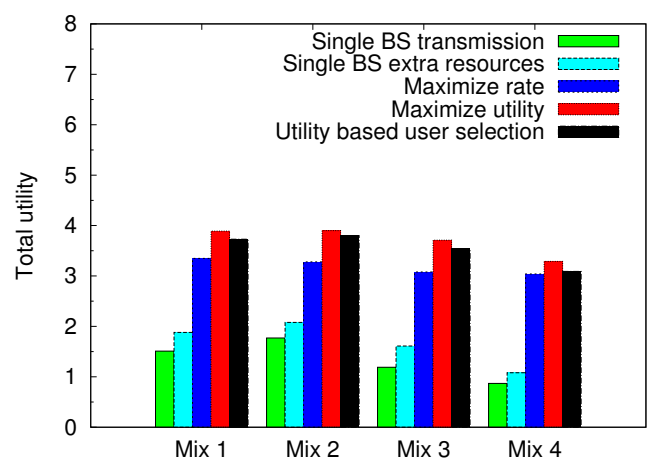

Fig. 10. Avg. over backhaul capacities of the total utility, all users at the cell edge

The results for all users located at the cell edge are summarized in Fig. 10. The gain of CoMP, as compared to single BS transmission, is significantly higher when all the users are located at the cell edge. The increase in the average total utility with maximize utility as compared to maximize rate is $15.9 \%$, which is slightly lower than for users spread out between the BSs. The highest average increase of $20.2 \%$ is reached for traffic mix 3, and the lowest, $7.9 \%$ for traffic mix 4 . When all users are located at the cell edge, single BS transmission is, for most shadow fading values, not sufficient to provide a high utility for hard real-time applications. Maximize utility, therefore, often selects users of hard real-time applications, and also adaptive applications. Also here, maximize utility may increase the total utility more for individual traffic mixes and backhaul capacities, in some cases as much as $47.1 \%$, and for some shadow fading sets, the increase even exceeds $117.3 \%$. The results of single BS extra resources is closer to the ones for single BS transmission than the ones for CoMP.

\section{CONCLUSION}

In this paper, we present simulation results of user selection for joint processing CoMP under limited backhaul capacity. User experience, represented by the utility of Internet application types, is evaluated as a criterion for user selection. Maximize utility, a user selection scheme with the objective to maximize the sum of the application utilities, is compared to maximize rate, which has the objective to maximize the sum data rate. As the computational complexity of maximize utility is high, we also propose and evaluate a heuristic algorithm with lower complexity, utility based user selection.

Simulations are conducted for two user scenarios and under a range of traffic mixes, backhaul capacities and shadow fading values. The results show that the average total utility is higher for maximize utility and utility based user selection than for maximize rate for all the tested parameter values. The existence of users of hard real-time and adaptive applications in a traffic mix seems to have a larger impact on the results, than the proportions of elastic applications. The gain of using maximize utility or utility based user selection is on average $15.9-17.6 \%$, and, for certain combinations of traffic mix, backhaul capacity and shadow fading values the gain is considerably higher, in some cases even twice as high. The average gain of using maximize utility or utility based user selection over maximize rate may become even higher if there were more users to chose from when users are selected for CoMP. In order to limit the computational complexity, we limited the number of users in our study. User selection needs to be combined with user scheduling on smaller time scales. This is a topic we intend to study further.

\section{ACKNOWLEDGMENT}

This work is part of Project 2009-4555 Dynamic Multipoint Wireless Transmission which is financed by the Swedish Research Council.

\section{REFERENCES}

[1] L. Shi, C. Liu, and B. Liu, "Network utility maximization for tripleplay services," Computer Communications, vol. 31, pp. 2257-2269, June 2008.

[2] J. Sachs, M. Prytz, and J. Gebert, "Multi-access management in heterogeneous networks," Wireless Personal Communications, vol. 48, pp. 732, January 2009.

[3] S. Shenker, "Fundamental design issues for the future Internet," IEEE Journal of Selected Areas in Communication, vol. 13, pp. 1141-1149, September 1995.

[4] 3GPP, "TR 36.814-900 3rd Generation Partnership Project; Technical Specification Group Radio Access Network; Further Advancements for E-UTRA Physical Layer Aspects (Release 9);"' March 2010.

[5] D. Gesbert, S. Hanly, H. Huang, S. Shamai, O. Simeone, and W. Yu, "Multi-cell MIMO cooperative networks: A new look at interference," IEEE Journal on Selected Areas in Communications, vol. 28, pp. 1380 - 1408, December 2010.

[6] R. Irmer, H. Droste, P. Marsch, M. Grieger, G. Fettweis, S. Brueck, H.-P. Mayer, L. Thiele, and V. Jungnickel, "Coordinated multipoint: Concepts, performance, and field trial results," IEEE Communications Magazine, vol. 49, pp. 102-111, February 2011.

[7] R. Apelfröjd, M. Sternad, and D. Aronsson, "Measurement-based evaluation of robust linear precoding for downlink CoMP," IEEE International Conference on Communications, ICC, Ottawa, Canada, pp. 57495755, June 2012.

[8] A. Klockar, C. Botella, T. Svensson, A. Brunstrom, and M. Sternad, "Utility of joint processing schemes," The Seventh International Symposium on Wireless Communication Systems (ISWCS), September 2010.

[9] A. Papadogiannis, H. Bang, D. Gesbert, and E. Hardouin, "Efficient selective feedback design for multicell cooperative networks," IEEE Transactions on Vehicular Technology, vol. 60, pp. 196 - 205, January 2011.

[10] P. Hande, S. Zhang, and M. Chiang, "Distributed rate allocation for inelastic flows," IEEE/ACM Transactions on Networking, vol. 15, pp. 1240-1253, December 2007. 